\title{
TOPOLOGICAL FEATURES OF INVISCID FLOWS
}

\author{
ROBERT GHRIST \& RAFAL KOMENDARCZYK \\ School of Mathematics \& $C D S N S$ \\ Georgia Institute of Technology \\ Atlanta, GA 30332-0160, USA \\ E-mail: ghrist@math.gatech.edu6r rako@math.gatech.edu
}

\begin{abstract}
The Euler equations for an incompressible inviscid fluid in dimension three possess a wealth of topological phenomena woven into the dynamical and geometric properties of the fluid. Focusing first on steady Euler fields, we outline known results, giving special attention to the Beltrami fields and the contemporary topological techniques required to elucidate their dynamical features. We also propose a topological perspective for understanding the global dynamics of the Euler equations on the space of velocity fields.
\end{abstract}

\section{Introduction to Geometric Euler Flows}

The behavior of the velocity field, $u$, of a perfect, inviscid, incompressible fluid is governed by the Euler equations:

$$
\frac{\partial u}{\partial t}+\nabla_{u} u=-\nabla p \quad ; \quad \operatorname{div}(u)=0 .
$$

Here, $p$ is a real-valued (time-dependent) function measuring pressure. On Euclidean 3 -space, the term $\nabla_{u} u$ is also written $(u \cdot \nabla) u$. These equations are, for the obvious reasons, usually considered only on a Euclidean domain. However, since we are interested in taking a topological perspective, there is no reason not to consider these equations in their most general setting.

Let $M$ denote a manifold of dimension $n$ (we will usually consider the case $n=3$ for simplicity). For any time-dependent velocity field $u$ on $M$, all of the terms in (1) save the first require some addition structure in order to compute. Thus, choose a Riemannian metric $g$ on $M$ : this $g$ is a smoothly-varying inner-product on each tangent space to $M$. Via $g$, one 
may then compute all the standard quantities of vector calculus, including the gradient of $p: M \rightarrow \mathbb{R}$ and the covariant derivative $\nabla_{u} u$ of the vector field $u$ in the direction $u$. In addition, to talk of divergence-free fields, one needs a notion of volume on $M$ : this too can be chosen as some density (a volume form $\mu$ to be precise) which may or may not be equal to that derived from the metric $g$. For the reader not familiar with the basics of differential geometry in the context of fluids, we would recommend selected passages of [4] or [1].

The wealth of topological phenomena that arise within the Euler equations cannot be under-estimated. We restrict to the goal of convincing the reader that cutting-edge topological techniques are not only efficacious but crucial for understanding even the most basic types of fluid flows. The reader who thirsts for more will find a nearly inexhaustible supply of interesting ideas and results in the recent excellent monograph of Arnold and Khesin [4].

The Euler equations are difficult enough on the tame Euclidean 3-space we so often work in: even the existence of solutions for all time is unknown and not at all clear. In order to get any concrete results for arbitrary geometric manifolds, we must begin modestly. For almost the entire remainder of this work, we restrict to the class of steady solutions to the Euler equation: hence,

$$
\nabla_{u} u=-\nabla p \quad ; \quad \operatorname{div}(u)=0,
$$

where neither $u$ nor $p: M \rightarrow \mathbb{R}$ is time-dependent.

While the reader (and the authors!) may be most interested in proving results about the case where $M=\mathbb{R}^{3}$ and $g$ is the Euclidean metric, it is nevertheless both interesting and instructive to see how steady Euler flows manifest themselves in other geometric contexts.

Example 1.1 [Hopf field] Consider the 3-sphere, denoted $S^{3}$, which consists of all points in $\mathbb{R}^{4}$ a unit distance from the origin. Via stereographic projection, this 3-manifold is equivalent to $\mathbb{R}^{3}$ with an added "point at infinity." The round metric on $S^{3}$ is that inherited by it as a subset of Euclidean $\mathbb{R}^{4}$, much in the same manner as we would describe the geometry of the round 2-sphere $S^{2} \subset \mathbb{R}^{3}$. The simplest example of a steady Euler field on the round $S^{3}$ is that given by the Hopf field, pictured in Figure 1. One may realize this field in Euclidean coordinates on $\mathbb{R}^{4}$ via

$$
X_{H}:=\left\{\begin{array}{cc}
\dot{x}=-y & ; \quad \dot{z}=-w \\
\dot{y}=x & ; \quad \dot{w}=z
\end{array}\right.
$$

These equations correspond to the motion of two identical uncoupled simple harmonic oscillators at a fixed energy level. Thus, every orbit of the flow is periodic, or closed. Indeed, this is an extraordinary flow: every orbit is a geodesic on $S^{3}$, and the entire flow is symmetric. 


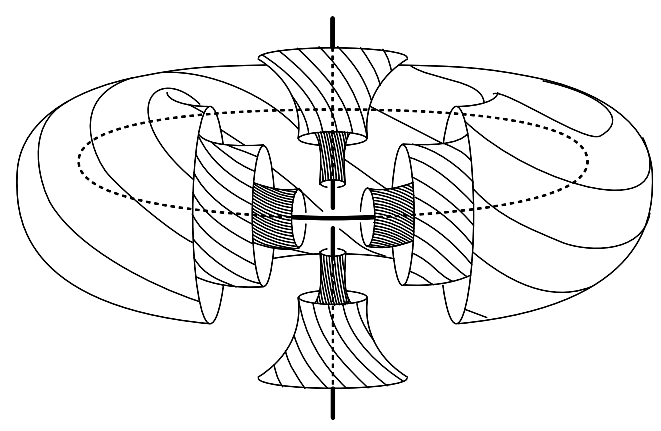

Figure 1. The Hopf flow on $S^{3}=\mathbb{R}^{3}+\{\infty\}$ is filled with closed unknotted flowlines which fill a 1-parameter family of invariant tori limiting onto a pair of circles.

Note that this fluid flow is filled with flowlines that are (1) closed, and (2) unknotted. It is a remarkable feature of inviscid fluids that both of these properties persist in some vestigial form no matter how one deforms the metric on $S^{3}$ or the conserved volume or the dynamics of the steady flow:

Theorem 1.2 [11] Every non-vanishing solution to (2) on any Riemannian $S^{3}$ possesses a closed, unknotted flowline, assuming that the vector field and metric are sufficiently smooth. ${ }^{1}$

Such is not the case for non-Euler vector fields [27, 28]: being a steady solution to the Euler equations is restrictive. This result hints that there are features of steady Euler flows which are entirely topological, independent of the geometry of the underlying manifold.

\section{Integrable Flows}

One's first thought is that the problem of analyzing steady Euler flows on a Riemannian manifold is trivial: consider the following adaptation of the Bernoulli theorem (cf. Arnold's theorem [2]).

'Not-quite-true' Theorem: Given u a steady non-vanishing Euler flow on $M$ a compact 3-manifold without boundary. If everything is of smoothness class $C^{\omega}$ (real-analytic), then $M$ is filled almost everywhere by invariant tori.

'Almost-correct' Proof: Transform Equation (2) via a standard vector-calculus identity into the Bernoulli form of the equation,

$$
u \times(\nabla \times u)=-\nabla H,
$$

\footnotetext{
${ }^{1}$ Real analytic, or $C^{\omega}$, is the simplest set of sufficient conditions.
} 
where $H: M \rightarrow \mathbb{R}$ is the Bernoulli function and $\nabla \times u$ is the vorticity field of the fluid. Take the dot-product of both sides of the equation with $u$. Since the left-hand side is the scalar triple product of $u$ with itself and with $\nabla \times u$, we obtain $u \cdot \nabla H=0$. In other words, $H$ is constant along orbits of $u$. Now, thanks to the Inverse Function Theorem, the level sets of $H$ in $M$ are generically smooth, closed, invariant submanifolds of dimension two. As the vector field $u$ is non-vanishing, each level set $H^{-1}(c)$ must have Euler characteristic zero: this implies (via the Classification Theorem for Surfaces) that $H^{-1}(c)$ is either a torus or a Klein bottle. However, being oriented by the vector $\nabla H$, the non-degenerate level sets of $H$ are invariant tori. Since everything is $C^{\omega}$, at most a finite number of level sets of $H$ can be degenerate, and these must be of codimension strictly greater than zero.

QED

If this proof were correct, then it would imply that, under the stated hypotheses, non-vanishing steady Euler flows would be very rare beasts. They would simply not exist on most three-dimensional manifolds! Indeed, any closed manifold which can be outfitted with a hyperbolic geometry cannot be filled almost-everywhere with tori, no matter what the actual geometry of the manifold is. All such steady Euler flows would qualitatively look like the Hopf flow of Figure 1, and would not be terribly interesting.

The mistake, of course, lies in the assertion that the function $H: M \rightarrow \mathbb{R}$ is not a constant function. In that (and only in that) case ${ }^{2}$ the conclusions fail since the level set of $H$ is all of $M$. Notice that if $H$ is a constant on $M$, then $u \times(\nabla \times u)$ is necessarily zero: $u$ is parallel to its curl.

The true result that can be proved (the modern formulation of the Bernoulli Theorem, due to Arnold ${ }^{3}$ ) is the following:

Theorem 2.1 [2] Given u a steady non-vanishing Euler flow on $M$ a compact 3-manifold without boundary. If everything is of smoothness class $C^{\omega}$ (real-analytic), then either (1) $M$ is filled almost everywhere by invariant tori; or (2) $u$ is an eigenfield of the curl operator.

This one case will occupy the remainder of our attention.

\section{Beltrami Fields}

Definition 3.1 A volume-preserving vector field $u$ is Beltrami if it is an eigenfield of the curl operator: i.e., if $\nabla \times u=\lambda u$ for some $\lambda \in \mathbb{R}$.

\footnotetext{
${ }^{2}$ This is where the $C^{\omega}$ assumption is critical.

${ }^{3}$ Arnold actually proves quite a bit more than stated - his proof concerns arbitrary integrable Hamiltonian systems and consists in showing that the velocity and vorticity fields commute. It is curious that, for three-dimensional vector fields, one does not require that the flow be volume-preserving: only an integral of motion is needed.
} 
Example 3.2 [Hopf field] The Hopf field on $S^{3}$ is an example of a Beltrami field on the round $S^{3}$ with eigenvalue 1 . Notice that, although it is Beltrami, the dynamics of the field still satisfy the conclusions of Arnold's Theorem.

Example 3.3 [ABC fields] The most historically influential example of a Beltrami field is the following:

$$
\dot{x}=A \sin z+C \cos y \quad ; \quad \dot{y}=B \sin x+A \cos z \quad ; \quad \dot{z}=C \sin y+B \cos x
$$

In this equation, $A, B$, and $C$ are parameters. For all such parameter values, one has a Beltrami field on the Euclidean $\mathbb{R}^{3}$, or, by quotienting out the periodic boundary conditions, on the three-dimensional torus $T^{3}$. For parameter values satisfying $A^{2}>B^{2}+C^{2}$, the field is non-vanishing. As displayed in Figure 2, the dynamics can be rather complicated - one appears to have regions of chaotic, non-integrable flow.
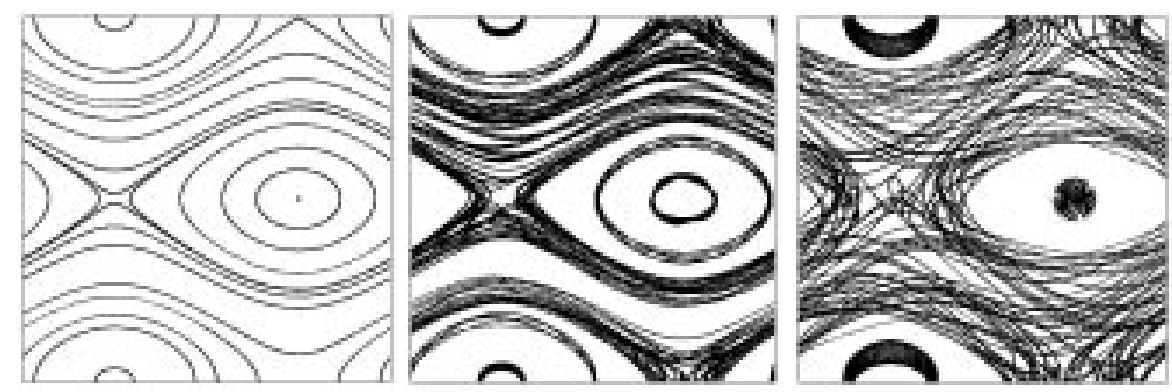

Figure 2. The ABC fields represented in a fundamental domain of $\mathbb{R}^{3}$, projected onto a square. The dynamics display integrable behavior (left) when one of the parameters is zero; increasing the parameter (middle,right) reveals increasingly non-integrable dynamics.

Despite the initial appearance of being the "exceptional" steady Euler fields, Beltrami fields turn out to be surprisingly important objects [4, 31]:

1. As already mentioned, Beltrami fields are the only steady solutions to the Euler equations which may exhibit "chaotic" Lagrangian dynamics.

2. The $L^{2}$-energy of a fluid $u$ is the integral of $\|u\|^{2}$ over the manifold. If one considers this functional restricted to all vector fields $\phi_{*} u$ obtained from $u$ by deforming it with a volume-preserving diffeomorphism $\phi$, then the Beltrami fields are critical points of this functional [3].

3. Every Beltrami field with non-zero eigenvalue has a non-vanishing helicity, a measure of average asymptotic linking of flowlines [3] which forms a lower bound on the $L^{2}$-energy of the fluid.

4. Given a perfectly-conducting fluid, the Beltrami fields yield steady solutions to the MHD equations linking the fluid flow and its induced 
magnetic field. This is an important class of steady plasmas. In ideal MHD, Beltrami fields are thus called force-free fields [8].

Despite their relative importance, there have been relatively few rigorous results about the dynamics of Beltrami fields. Nearly everything known about their dynamics comes from either numerical experiments [9] or from applying Melnikov analysis to near-integrable examples [7, 20], a very narrow band of fields.

There are numerous open problems concerning the dynamics and topology of Beltrami fields.

Question 3.4 Does every Riemannian 3-manifold admit a Beltrami field without fixed points?

Every three-manifold admits volume-preserving vector fields without fixed points: the question is whether any of these can be Beltrami. The spectral theorem applied to the curl operator (whose square is the Laplacian) quickly yields the existence of infinitely many Beltrami fields on every Riemannian 3-manifold. The analytic approach provides no control over the existence of fixed points, however. We believe that when the manifold is sufficiently negatively curved, Beltrami fields always possess fixed points.

Question 3.5 Given a Beltrami field on a closed 3-manifold, how many periodic orbits must exist?

This problem is a distant cousin of the Arnold Conjecture in the setting of fluids (see [30] for an introduction to the Arnold Conjecture in symplectic dynamics). One's first guess is that every Beltrami field must have infinitely many periodic orbits. However, this is not true: by deforming the round 3sphere into something ellipsoidal, one can perturb the Hopf flow into a Beltrami field which has but two periodic orbits. It is an open conjecture that the minimum for the 3-sphere (in any metric) is two, and that any more than two implies infinitely many. A far-reaching tool for this conjecture is the symplectic field theory of [10].

Question 3.6 Are there any parameter values for which the ABC fields possess a closed orbit on $\mathbb{R}^{3}$ ?

Such orbits definitely do not exist when any of the parameters $A, B$, or $C$ is equal to zero. In $\S 5$, we outline a program for finding such an orbit.

Question 3.7 Characterize the topology of the flowlines of Beltrami fields. What kinds of knots must/can be traced out by periodic flowlines?

Several partial results will be stated in $\S 6$.

Question 3.8 Under what circumstances is a Beltrami field forced to possess a closed flowline which is hyperbolic? That is, the eigenvalues of the linearization of the Poincaré return map are not equal to one. 
This problem is, thanks to [19], a route to establishing hydrodynamic instability for steady Euler fields. We comment more on this problem in $\S 7$.

\section{An Introduction to Contact Topology and Dynamics}

Surprisingly, understanding the dynamics of Beltrami fields seems to require a foray into some rather esoteric-looking branches of three-dimensional topology. But a self-enforced exile to the furthest abstraction is rarely permanent and certainly not without reward. Most every question is $\S 3$ can be approached via techniques involving contact structures.

\subsection{CONTACT STRUCTURES AND FORMS}

For our purposes, a contact structure on a 3-manifold $M$ is a certain type of smoothly-varying plane field $\xi$. Just as a vector field assigns a vector to each tangent space of $M$, a plane field assigns some 2-dimensional subspace to each tangent space. For example, given a vector field $X$ on a Riemannian 3 -manifold $M$, the 2-D subspace orthogonal to $X$ in each tangent space define a plane field on $M$.

While any smooth vector field can always be "integrated" into a collection of smooth curves tangent to the field, such is not the case for a general plane field. Only in very rare circumstances can one stitch together the planes (like scales on a fish) to fill $M$ with two-dimensional sheets tangent to the planes. Most often, the planes twist in such a way as to break the integrability. Occasionally, there can be found plane fields which twist so much as to be nowhere-integrable, not even at a single point.

Definition 4.1 A contact structure on a 3-manifold $M$ is a smoothlyvarying plane field $\xi$ which is completely non-integrable.

Two more precise definitions can be formulated:

1. $\xi$ is a contact structure iff for any pair of locally non-zero vector fields $U, V$ tangent to $\xi$, their Lie bracket $[U, V]$ is nowhere tangent to $\xi$.

2. $\xi$ is a contact structure iff it can be written [locally] as the kernel of a contact form - a differential 1-form $\alpha$ satisfying

$$
\alpha \wedge d \alpha \neq 0
$$

That these definitions are all equivalent is really the Frobenius Theorem [1]. The two above definitions are most useful in computations, while the "twisted plane field" definition is perhaps the most readily grasped. The best way to "visualize" a contact form is in terms of the kernels of $\alpha$ and $d \alpha$. On each three-dimensional tangent space, the kernel of $\alpha$ is a twodimensional plane: the contact element. The 2 -form $d \alpha$ has a 1-dimensional 
kernel consisting of those vectors which annihilate $d \alpha$ when plugged into the first slot. Equation (6) translates into saying that $\alpha$ is a contact form iff these subspaces are never tangent, see Figure 3(left).
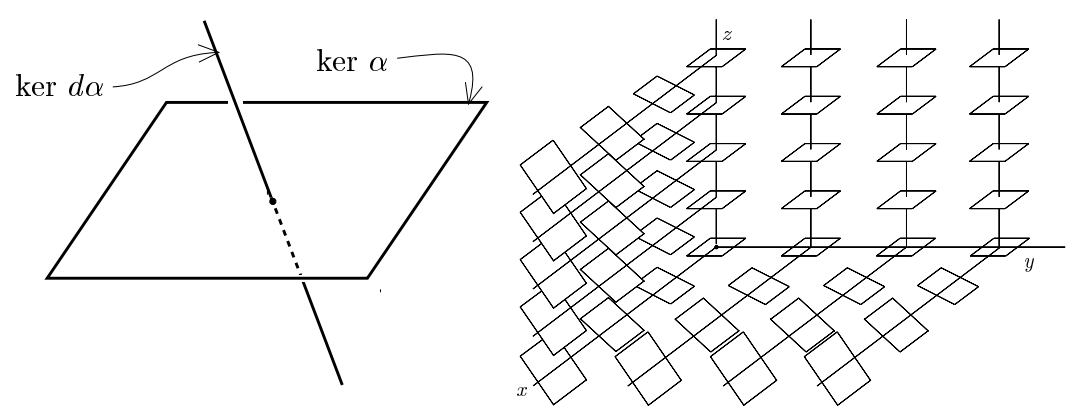

Figure 3. (left) Visualizing a contact form at a tangent space; (right) Every contact structure is locally equivalent to the kernel of $d z+x d y$.

Example 4.2 [Standard contact structure on $\mathbb{R}^{3}$ ]

Consider the 1-form

$$
\alpha_{1}:=d z+x d y .
$$

where $\{d x, d y, d y\}$ are the duals (co-vectors) to the standard basis vectors in $\mathbb{R}^{3}$. Since $d \alpha_{1}=d x \wedge d y$, we have that $\alpha_{1} \wedge d \alpha_{1}=d z \wedge d x \wedge d y$ which is nowhere zero (a volume form). Thus, the kernel of $\alpha_{1}$ is a contact structure. This kernel is defined by solving $\alpha_{1}=0$ to obtain $d z / d y=-x$. That is, the slope of the plane field is given by $-x$ : see Figure 3(right). If an observer walks along any $\mathrm{x}$-axis, the plane field $\xi$ twists counter-clockwise.

The Darboux theorem states that the standard contact form is a "building block" for any contact form on any 3-manifold, i.e., any contact form under appropriate change of coordinates can be locally expressed by (7).

Example 4.3 [Standard contact structure on $S^{3}$ ] Recall Example 1.1 of the Hopf field on the round $S^{3}$. The plane field orthogonal to this vector field is a contact structure. One may check this by recalling the vector field $X_{H}$ of (3) and defining the 1 -form $\alpha_{2}$ via $\alpha_{2}(V):=X_{H} \cdot V$. One readily shows that $\alpha_{2}=\frac{1}{2}(x d y-y d x+z d w-w d z)$, and that this is indeed a contact form satisfying (6) on $S^{3}$.

\subsection{REEB FIELDS}

Example 4.3 gives several hints that contact structures are dynamically interesting objects. First, being related to the Hopf field, this contact structure is related to the global topological features of the three-sphere. Second, 
the Hopf field actually lies in the kernel of $d \alpha_{2}$, as a simple computation reveals. It thus follows (from the Cartan formula) that the flow of the Hopf field preserves the contact form.

Indeed, if we consider an arbitrary contact form $\alpha$, the "cartoon" representation of Figure 3(left) indicates that the kernel of $d \alpha$ provides a "natural" or "canonical" direction on each tangent space. Choosing a non-zero vector in each such line yields a vector field on $M$ whose dynamics ought to be related to the geometry of the contact structure. Indeed, this observation naturally leads one to the following definition:

Definition 4.4 The Reeb field associated with a contact form $\alpha$ is defined as the unique vector field $X$ satisfying

$$
\text { i) } \quad d \alpha(X, \cdot)=0 \text { and ii) } \alpha(X)=1 .
$$

$A$ Reeb-like field is one for which we relax the second condition to $\alpha(X)>$ 0 .

The flow of a Reeb field leaves the contact structure and the contact form invariant. They are always volume-preserving for the natural volume form $\alpha \wedge d \alpha$.

Example 4.5 [Reeb fields] The Reeb field associated with the local contact form $\alpha_{1}$ of Example 4.2 is the vertical field $\frac{\partial}{\partial z}$. The Reeb field associated with the contact form $\alpha_{2}$ orthogonal to the Hopf field is precisely the Hopf field $X_{H}$.

It is by no means a coincidence that the definition of a contact structure as a plane field which "everywhere twists" and the intuition of a rotational Beltrami field as a vector field which "everywhere rotates about itself" are so similar. The following correspondence asserts roughly that all Beltrami fields are Reeb fields, and vice versa (up to reparametrizations by time, which changes none of the topological features):

\begin{tabular}{|c|c|}
\hline $\begin{array}{c}\text { non-singular rotational } \\
\text { Beltrami fields } \\
\text { for all metrics, } \\
\text { all non-zero scalings }\end{array}$ & $\Leftrightarrow \quad \begin{array}{c}\text { all Reeb fields } \\
\text { for all contact forms, } \\
\text { all non-zero scalings }\end{array}$ \\
\hline
\end{tabular}

One direction is trivial: the orthogonal plane field associated with any Beltrami field is always a contact structure. To go in the other direction, one begins with a Reeb-like field and constructs a metric for which the field is Beltrami (see [12] for details and simple proof).

The category of Beltrami fields is geometric and rigid. But, after collecting all Beltrami fields for all metrics together, the resulting class is the 
set of Reeb fields - a decidedly topological category. This allows one to prove a number of non-trivial result about Beltrami fields by relaxing the metric constraints and treating them as topological objects.

One immediate application is to the problem of existence of non-singular Beltrami fields (Question 3.4). According to [29], every 3-manifold admits a contact structure. Any Reeb field associated with this yields a Beltrami field; thus,

Corollary 4.6 Every 3-manifold admits a non-singular Beltrami flow for some Riemannian structure.

Contact perspectives also allow us to construct examples of Beltrami fields on higher dimensional manifolds, e.g., analogues of the ABC fields (5) on the 5-torus $T^{5}[21]$.

\subsection{HOFER'S THEOREM AND OVERTWISTED STRUCTURES}

A compelling reason for understanding contact structures and their topological properties comes from recent work of Hofer and collaborators [25, 26, 10]. The programme outlined in [10] indicates that the topological properties of a contact structure are delicately entwined with the dynamical properties of the Reeb fields associated with that contact structure, much in the same way that the topology of a manifold can be recovered via the dynamics of a gradient field. The first hint of this relationship appears in the paper [25] which finds that certain types of contact structures are forced to have periodic orbits in their Reeb fields. Understanding and applying this result requires a deeper understanding of the topological classification of contact structures.

To summarize roughly the past twenty years of contact topology in one idea: the best way to analyze a contact structure $\xi$ on $M$ is to consider how $\xi$ intersects various two-dimensional discs in $M$. Dynamical properties of these intersections correlate to dynamical properties of Reeb fields.

Definition 4.7 Given a contact structure $\xi$ and a disc $D$ embedded in $M$, the characteristic foliation, $D_{\xi}$, is the set of curves carved out on $D$ by intersecting the planes of $\xi$ with the tangent planes of $D$ in $M$.

Definition 4.8 The contact structure $\xi$ is overtwisted if there exists an embedded disc $D$ in $M$ whose characteristic foliation $D_{\xi}$ contains a limit cycle (an attracting periodic orbit): see Figure 4. If no such disc exists, then $\xi$ is called tight.

Example 4.9 [Tight vs. overtwisted] The standard contact structures for $\mathbb{R}^{3}$ and $S^{3}$ given in Examples 4.2 and 4.3 are tight. A concrete example of an overtwisted structure on $\mathbb{R}^{3}$ is that given by the form (in polar coordinates):

$$
\alpha_{3}=\cos (r) d z+r \sin (r) d \theta .
$$




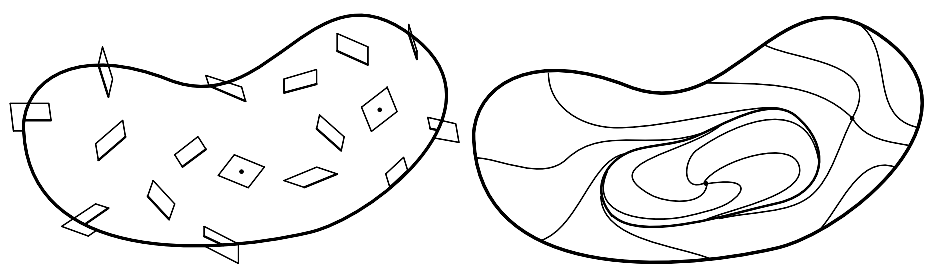

Figure 4. A contact structure slices a disc into curves and fixed points (of tangency). An overtwisted contact structure carves a limit cycle into some disc.

For this contact form, the slope of the contact form is given by $d z / d \theta=$ $-r \tan r$; the planes "flip" over many times, in contrast to Example 4.2.

Hofer's theorem states the surprising result that a limit cycle in the dynamics of the contact planes (intersecting a disc) implies the existence of a periodic orbit in the transverse Reeb dynamics:

Theorem 4.10 [25] Any Reeb field for an overtwisted contact structure on a closed 3-manifold $M$ possesses a periodic orbit (some multiple of which can be shrunk to a point in $M$ ).

This theorem, a tour-de-force of symplectic geometry and non-linear analysis, is essentially the only available global tool for analyzing the dynamics of arbitrary 3-D Beltrami fields (though see [10]).

\section{Existence of Closed Orbits}

\subsection{CLOSED ORBITS}

Hofer's techniques, combined with the correspondence between Reeb and Beltrami fields, quickly yields existence results for closed orbits of arbitrary Beltrami fields on certain 3-manifolds. We state a few examples.

Theorem 5.1 [12, 13] Let $u$ be a steady non-vanishing Euler flow of class $C^{\omega}$ (or, if restricting to Beltrami fields, $C^{2}$ suffices). Then u possesses a closed flowline in the following cases:

On any Riemannian 3-sphere, for any such u;

On any Riemannian solid torus, for any u tangent to the boundary;

On any Riemannian 3-torus, for any $u$ which is homotopically nontrivial. $^{4}$

The proofs of these results all have three parts: (1) proving in the case of an integrable Euler field (this requires the $C^{\omega}$ assumption); (2) determining

\footnotetext{
${ }^{4}$ Homotopically trivial means that one can rotate the vectors of $u$ so that they all point in the same direction.
} 
which Beltrami fields are tight, and which are overtwisted; (3) using Hofer's Theorem in the overtwisted cases and some clever techniques for the tight cases, which depends on the manifold. The genuinely difficult case is that of the solid torus $D^{2} \times \mathbb{R}$; this also is the case most closely tied to applications in plasma dynamics. We elaborate on the solid torus below.

\subsection{A 3-D INDEX}

Consider a non-vanishing Beltrami field $u$ on a tube $D^{2} \times \mathbb{R}$ which is periodic in the third variable and tangent to the boundary of the tube. Choose any convenient cross-sectional disc $D$, and project the vector field $u$ orthogonally onto $D$. This generates a vector field $u^{\perp}$ on $D$ tangent to the boundary which generically has a finite number of fixed points of type source/sink or saddle. The standard Euler-Poincaré index theory says that the sum of the indices of the fixed points on $D$ ( +1 for sources/sinks and -1 for saddles) equals the Euler characteristic of $D$, which is 1 . Such arguments are often used to find fixed points or periodic orbits in 2-D flows.

However, for $D$ and $u^{\perp}$ arising from a Beltrami field, there is a vestige of this 2-D index theory. Consider the fixed points of $u^{\perp}$ on $D$ : these are the points at which $u$ is orthogonal to $D$. There is thus an additional sign depending on whether $u$ points to the "right" or the "left" of the disc (see Figure 5).
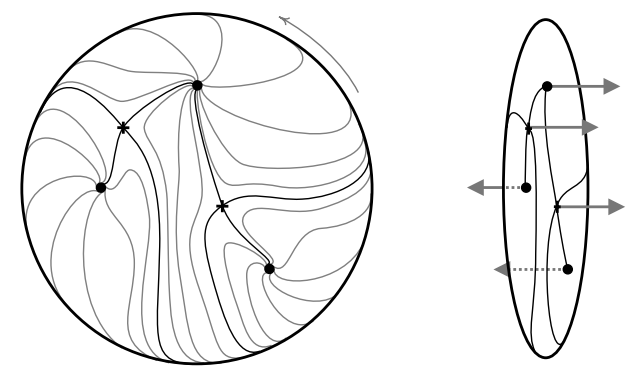

Figure 5. Projecting a Beltrami field to a cross-sectional disc yields a vector field with weighted index -2 . The Beltrami field thus has a closed orbit in $D \times \mathbb{R}$.

Theorem 5.2 [15] With $D, u^{\perp}$ as above, compute the Euler-Poincaré index of the vector field $u^{\perp}$ on $D$, weighting each term with a $(+)$ if the vector field $u$ points to the right and (-) if it points to the left of $D$. If this weighted index is not \pm 1 , then the Beltrami field $u$ has a closed flowline in $D^{2} \times \mathbb{R}$.

The proof of this result is to use the stated information about $u^{\perp}$ to reconstruct the contact planes orthogonal to $u$ on $D$. Through topologi- 
cal techniques, the weighted index computation indicates that the contact structure on $D \times \mathbb{R}$ is overtwisted. One then applies Hofer's techniques.

This is really oblique: one knows nothing about the field $u$ except that (1) it is Beltrami; and (2) it is orthogonal to some disc $D$ at certain points with certain behavior. Nothing else is known about $u$ away from the disc $D$. Genuinely two-dimensional data yields a genuinely three-dimensional implication. Of course, the result holds in an arbitrary metric, and the modified index is independent of the choice of disc $D$. It is remarkable that the chosen disc $D$ might not intersect the closed orbit it detects. Even so, deforming the metric and the Beltrami field by an arbitrary amount off of the disc will not destroy the closed orbit.

This is a prime example of a topological result: it is stable with respect to arbitrarily large perturbations.

\section{Knots}

In dimension three, periodic flowlines are embedded closed curves: knots, which together form a link of closed curves. There is a long beautiful string of theory about the relationships between the dynamics of a threedimensional flows and the topology of the link of closed orbits (see, e.g., $[5,23]$ and references therein).

By Theorem 2.1, it is necessary to partition our discussion of knot types into two classes: the integrable case and the Beltrami case.

\subsection{INTEGRABLE FLOWS}

As hinted at by Example 1.1, the knot types present in an integrable vector field are especially simple. Since almost all of the orbits are constrained to lie on a torus, one expects a certain rigidity.

Definition 6.1 The connected sum of two knots $K$ and $K^{\prime}$ is the knot obtained by splicing the two together as in Figure 6(left). Given a knot K, the cables of $K$ are those knot types which can be drawn as simple closed curves on the surface of a torus having $K$ at its core (Figure 6(right)). The torus knots are those knot types which are the cables of the unknot.

The following theorem classifying knots of integrable fields has a convoluted history. It was first stated (with a slightly stronger assumption) by Fomenko and Nguyen [16]. Casasayas et al. [6] observed that their result follows effortlessly from earlier work of Wada [33] and Morgan [32].

Theorem 6.2 Every closed orbit in a non-vanishing $C^{\omega}$ integrable vector field on $S^{3}$ is a knot obtained from the unknot by repeated cabling and connected sum. 

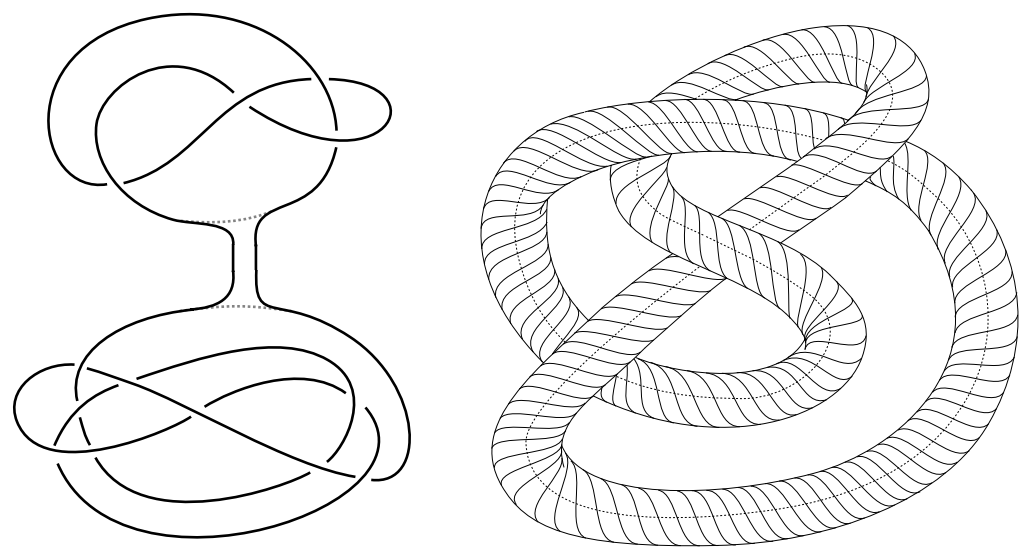

Figure 6. Connected sums (left) and a cable of the figure-eight knot (right).

Thus, the presence of a single figure-eight $\operatorname{knot}^{5}$ in a non-vanishing flow is enough to conclude that the flow is not integrable.

\subsection{BELTRAMI FIELDS}

Beltrami flows, in contrast to their integrable cousins, display the broadest possible variety of behavior with respect to flowline topology. The trick is in the correspondence between Reeb fields and Beltrami fields. Reeb fields, since they are topological in nature, are subject to all of the manipulations a topologist can muster: surgery, cut-and-paste, and the like. After a customized contact form is built, one invokes the correspondence theorem to realize this as a genuine Beltrami field for the appropriate Riemannian metric. Using a very delicate sequence of topological constructions, one can construct a Beltrami field which exhibits the most complex type of field line topology imaginable:

Theorem 6.3 [14] There exists a non-vanishing Beltrami field on a Riemannian 3-sphere 6 which possesses closed flowlines of all possible knot and link types.

The velocity field and the metric are all of class $C^{\omega}$ (real-analytic). These fields are by no means degenerate: any $C^{1}$-close vector field still possesses this set of knotted and linked flowlines. However, the construction most certainly does not yield a round (or Euclidean) metric.

\footnotetext{
${ }^{5}$ Or, indeed, any knot whose complement in $S^{3}$ admits a hyperbolic metric...

${ }^{6}$ The proof also works and is indeed easier for a Riemannian $\mathbb{R}^{3}$.
} 
Question 6.4 Is it possible for a smooth, steady Euler flow on Euclidean $\mathbb{R}^{3}$ to possess closed flowlines of all knot types?

The paper [22] gives sufficient conditions for this to occur in terms of existence of certain homoclinic or heteroclinic orbits. Whether such can be found in a steady Euler flow is not clear.

\section{Moving Beyond Steady Flows}

Given a dynamical system, the first task is to find and classify the fixed points. The next step of a qualitative analysis is to determine the existence and nature of any periodic orbits or connecting orbits in the global flow.

In the case of the Euler equations, one has a dynamical system on the space of all divergence-free vector fields. This survey has covered the basics of what can be said about the fixed points of this dynamical system: the steady Euler flows. ${ }^{7}$ The next logical step after finding the fixed points is to determine the stability of these solutions: this in itself is a delicate problem.

\subsection{INSTABILITY}

The problem of classifying the local behavior of fixed points is precisely the classical problem of hydrodynamic stability. Though there are manifold notions of how hydrodynamical stability or instability should be defined (see [17]), the goal is to determine whether nearby velocity fields (perturbations of a steady Euler field) remain in a vicinity of the steady field, or whether perturbations grow large.

The answer seems quite plain, but there appears to be no way to prove it in general. The conjecture is this: Almost every steady Euler field in dimension three is hydrodynamically unstable. It is a genuine challenge to make this a mathematically well-defined question. The first point is to settle on the type of instability one seeks: linear or nonlinear? energy or velocity norm? The paper [17] contains an excellent discussion of this issue.

The second order of business is to determine what possible sense one could ascribe to the phrase "almost every"? For the Beltrami fields, the idea of "almost every" is strained - for a generic Riemannian metric, the eigenvalues of the curl operator are simple, and there are thus a countable number of Beltrami fields, each of which is isolated in the space of volumepreserving vector fields. The only possible sense that "almost every" can take is "all but a finite number" or, equivalently, "for all sufficiently large

\footnotetext{
${ }^{7}$ We have restricted attention to the case of dimension three, but the contact topology perspective is efficacious in all odd dimensions. For even dimensions, the appropriate framework is that of symplectic topology, as pointed out by Ginzburg and Khesin [24]. There are some beautiful ways in which steady fluids in high dimensions have radically different behaviors depending on the parity of the dimension.
} 
eigenvalues." In this (most typical) setting, one most decidedly cannot perturb a Beltrami field in any manner which will yield a new Beltrami field (ignoring scalar multiples of the field).

In the particular case of the Euclidean metric, the symmetries provide for multiple eigenspaces of curl leading to parametrized families of Beltrami fields. The ABC fields are one such example (thus explaining the existence of three parameters) for the first eigenvalue of curl. In this context, one may restrict to such families and try to prove generic instability (as is done for most singular ABC fields in [18]).

The situation for steady integrable fields is much cloudier. Are such fields isolated in the space of volume-preserving vector fields? Can they appear in parametrized families? Clearly, in the presence of enough symmetry, whole families can arise: but it seems reasonable to expect that such families would have identical stability properties. Indeed, if a symmetric stable family could be found, this could provide a counter-example to the Instability Conjecture. We point out the following simple test case:

Question 7.1 Are the Hopf fields on the round 3-sphere hydrodynamically unstable?

As we see the situation, the best hope for establishing a universal result on hydrodynamic instability is to use the criteria of a fluid lyapunov number developed by Friedlander and Vishik using techniques from geometric optics.

Theorem 7.2 [19] Any steady Euler flow which possesses a hyperbolic periodic orbit is (linearly) hydrodynamically unstable (in $L^{2}$ norm).

The contact-topological techniques provide a partial answer. In the case of an overtwisted Beltrami field, the periodic orbit forced is not elliptic: it is either hyperbolic or degenerate This orbit is thus hyperbolic generically (among Reeb fields). In like vein, the symplectic field theory of [10] indicates that, generically in the space of Reeb fields on $T^{3}$ (even in the tight case!), there exist hyperbolic periodic orbits. When one includes the metric as a variable (thus working in the space of all Reeb fields as opposed to the discrete space of Beltrami fields for a fixed metric), one can rigorously prove that hyperbolic orbits (and thus instability) are generic.

\subsection{CONNECTIONS AND PERIODIC ORBITS}

The next step in a global analysis concerns simple recurrence. Recall that a homoclinic orbit for a dynamical system is one which approaches a certain fixed point asymptotically both forwards and backwards in time. A heteroclinic orbit (or "connection") is an orbit which approaches two distinct fixed points as time goes to $\pm \infty$. 
Question 7.3 Do the Euler equations admit any homoclinic or heteroclinic orbits?

There are no obvious topological obstructions to the existence of homoclinic orbits of the Euler equations. Heteroclinic orbits have what appear to be extraordinarily rigid obstructions. Assume that there is an orbit $u(t)$ of Equation (1) which limits to Beltrami fields $u^{+}$and $u^{-}$as $t \rightarrow \pm \infty$ respectively. Since the vorticity field is "frozen in" by the Euler flow, the vorticity fields $\nabla \times u(t)$ are all equivalent by volume-preserving diffeomorphisms, and, in the limits, $u^{+}$and $u^{-}$are themselves related: an unlikely situation apart from unusual symmetries.

Question 7.4 Classify the periodic orbits of the Euler equations in the space of volume-preserving vector fields.

Surely one could construct such a periodic orbit using a periodic pressure function $p_{t}: M \rightarrow \mathbb{R}$ : take for example $\mathbb{R}^{3}$ with linear flow and use a family of pressure functions $p_{t}(x, y, z)$ to rotate the velocity vectors uniformly. Examples would be most helpful for obtaining a clearer picture of the global structure of the Euler equations as a dynamical system on the space of volume-preserving vector fields.

\section{Acknowledgements}

The first author acknowledges the hospitality of the Isaac Newton Institute, and the support of the National Science Foundation (DMS-9971629).

\section{References}

1. Abraham, R., J. Marsden, and T. Ratiu. (1988) Manifolds, Tensor Analysis, and Applications. in Applied Math. Sci., 75, Springer-Verlag, Berlin, Heidelberg, New York.

2. Arnold, V. I. (1966) Sur la géométrie differentielle des groupes de Lie de dimension infinie et ses applications à l'hydrodynamique des fluides parfaits. Ann. Inst. Fourier, 16, 316-361.

3. Arnold, V. I. (1986) The asymptotic Hopf invariant and its applications. Sel. Math. Sov., 5, 327-345.

4. Arnold, V. I., and B. Khesin. (1998) Topological Methods in Hydrodynamics. Springer-Verlag, Berlin, Heidelberg, New York.

5. Birman, J., and R. Williams. (1983) Knotted periodic orbits in dynamical systems-I : Lorenz's equations. Topology, 22(1), 47-82.

6. Casasayas, J., J. Martinez Alfaro, and A. Nunes. (1993) Knotted periodic orbits and integrability. In Hamiltonian Systems and Celestial Mechanics, in Adv. Ser. Nonlinear Dynam., pp. 35-44. Number 4, World Sci. Pub., River Edge, NJ.

7. Chicone, C. (1996) A geometric approach to regular perturbation theory with an application to hydrodynamics. Trans. Amer. Math. Soc., 347(12), 4559-4598.

8. Childress, S., and A. Gilbert. (1995) Stretch, Twist, Fold: The Fast Dynamo. Springer-Verlag.

9. Dombre, T., U. Frisch, J. Greene, M. Hénon, A. Mehr, and A. Soward. (1986) Chaotic streamlines in the ABC flows. J. Fluid Mech., 167, 353-391. 
10. Eliashberg, Y., A. Givental, and H. Hofer. (2000) An introduction to symplectic field theory. ArXiV preprint math.SG/0010059.

11. Etnyre, J., and R. Ghrist. (1999) Stratified integrals and unknots in inviscid flows. Cont. Math., 246, 99-112.

12. Etnyre, J., and R. Ghrist. (2000) Contact topology and hydrodynamics I: Beltrami fields and the Seifert conjecture. Nonlinearity, 13, 441-458.

13. Etnyre, J., and R. Ghrist. (2001) Contact topology and hydrodynamics II: solid tori. To appear in Ergod. Theory \& Dyn. Sys., ArXiv preprint math.SG/9907112.

14. Etnyre, J., and R. Ghrist. (2000) Contact topology and hydrodynamics III: knotted flowlines. Trans. Amer. Math. Soc., 352, 5781-5794.

15. Etnyre, J., and R. Ghrist. (2001) An index for closed orbits in Beltrami fields. ArXiv preprint math.DS/0101095.

16. Fomenko, A. T., and T. Z. Nguyen. (1991) Topological classification of integrable nondegenerate Hamiltonians on isoenergy three-dimensional spheres. In Advances in Soviet Mathematics. (ed. A. T. Fomenko), pp. 267-296. 6, Amer. Math. Soc., Providence, RI.

17. Friedlander, S., and V. Yudovich. Instabilities in fluid motion. (1999) Notices Amer. Math. Soc., 46(11), 1358-1367.

18. Friedlander, S., A. Gilbert, and M. Vishik. (1993) Hydrodynamic instability for certain ABC flows. Geophys. Astrophys. Fluid Dynam., 73, 97-107.

19. Friedlander, S., and M. Vishik. (1992) Instability criteria for steady flows of a perfect fluid. Chaos, 3, 455-460.

20. Gautero, J.-L. (1985) Chaos lagrangien pour une classe d'écoulements de Beltrami. C. R. Acad. Sci. Paris Sér. II Méc. Phys. Chim. Sci. Univers. Sci. Terre, 301(15), $1095-1098$.

21. Ghrist, R. (2001) Steady nonintegrable high-dimensional fluids. To appear in Lett. Math. Phys. Preprint.

22. Ghrist, R., and P. Holmes. (1996) An ODE whose solutions contain all knots and links. Intl. J. Bifurcation and Chaos, 6(5), 779-800.

23. Ghrist, R., P. Holmes, and M. Sullivan. (1997) Knots and Links in ThreeDimensional Flows, Springer Lecture Notes in Mathematics 1654, Springer-Verlag, Berlin, Heidelberg, New York.

24. Ginzburg, V., and B. Khesin. (1994) Steady fluid flows and symplectic geometry. J. Geom. and Phys., 14, 195-210.

25. Hofer, H. (1993) Pseudoholomorphic curves in symplectizations with applications to the Weinstein conjecture in dimension three. Invent. Math., 114, 515-563.

26. Hofer, H., K. Wysocki, and E. Zehnder. (1998) The dynamics on three-dimensional strictly convex energy surfaces. Ann. of Math. (2), 148(1), 197-289.

27. Kuperberg, G. (1996) A volume-preserving counterexample to the Seifert conjecture. Comment. Math. Helv., 71(1), 71-97.

28. Kuperberg, G., and K. Kuperberg. (1994) Generalized counterexamples to the Seifert conjecture. Ann. of Math., 144, 239-268.

29. Martinet, J. (1971) Formes de contact sur les variétés de dimension 3, pp. 142-163. Springer Lecture Notes in Math. 209, Springer-Verlag.

30. McDuff, D., and D. Salamon. (1995) Introduction to Symplectic Topology. Oxford University Press, New York.

31. Moffatt, H. (1994) Topological aspects of the dynamics of fluids and plasmas. Advanced NATO ASI Series E: Applied Sciences, 218, Kluwer Academic Press.

32. Morgan, J. (1978) Nonsingular Morse-Smale flows on 3-dimensional manifolds. Topology, 18, 41-54.

33. Wada, M. (1989) Closed orbits of nonsingular Morse-Smale flows on $S^{3}$. J. Math. Soc. Japan, 41(3), 405-413. 